\title{
Food and beverage intakes according to physical activity levels in European children: the IDEFICS (Identification and prevention of Dietary and lifestyle induced health Effects In Children and infantS) study
}

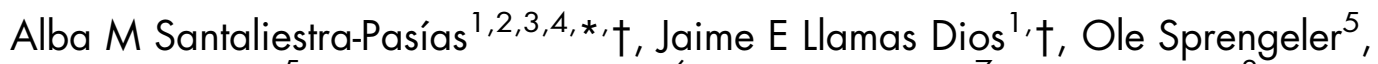 \\ Antje Hebestreit ${ }^{5}$, Stefaan De Henauw ${ }^{6}$, Gabriele Eiben ${ }^{7}$, Regina Felsö ${ }^{8}$, Fabio Lauria 9 , \\ Michael Tornaritis ${ }^{10}$, Toomas Veidebaum ${ }^{11}$, Valeria Pala ${ }^{12}$ and Luis A Moreno ${ }^{1,2,3,4}$ \\ 'GENUD (Growth, Exercise, Nutrition and Development) Research Group, Facultad de Ciencias de la Salud, \\ Universidad de Zaragoza, C/Domingo Miral s/n, Cp 50009 Zaragoza, Spain: ${ }^{2}$ Instituto Agroalimentario de Aragón \\ (IA2), Zaragosa, Spain: ${ }^{3}$ Instituto de Investigación Sanitaria Aragón (IIS Aragón), Zaragoza, Spain: ${ }^{4}$ Centro de \\ Investigación Biomédica en Red de Fisiopatología de la Obesidad y Nutrición (CIBERObn), Universidad de Zaragoza, \\ Zaragoza, Spain: ${ }^{5}$ Leibniz Institute for Prevention Research and Epidemiology - BIPS, Bremen, Germany: ${ }^{6}$ Department \\ of Public Health, Ghent University, Ghent, Belgium: ${ }^{7}$ Department of Public Health and Community Medicine, University \\ of Gothenburg, Gothenburg, Sweden: ${ }^{8}$ Department of Pediatrics, Medical Faculty, University of Pécs, Pécs, Hungary: ${ }^{9}$ Unit of \\ Epidemiology and Population Genetics, Institute of Food Sciences, National Research Council, Avellino, Italy: ${ }^{10}$ Research \\ and Education Institute of Child Health, Strovolos, Cyprus: ${ }^{11}$ National Institute for Health Development, Center of Health \\ and Behavioral Science, Tallinn, Estonia: ${ }^{12}$ Fondazione IRCCS Istituto Nazionale dei Tumori, Milan, Italy
}

Submitted 21 July 2017: Final revision received 23 November 2017: Accepted 2 January 2018: First published online 19 February 2018

\begin{abstract}
Objective: Physical activity (PA) levels and dietary habits are considered some of the most important factors associated with obesity. The present study aimed to examine the association between PA level and food and beverage consumption in European children (2-10 years old).

Design/Setting/Subjects: A sample of 7229 children (49.0\% girls) from eight European countries participating in the IDEFICS (Identification and prevention of Dietary and lifestyle induced health EFfects In Children and infantS) study was included. Moderateto-vigorous PA (MVPA) was assessed objectively with accelerometers. FFQ was used to register dietary habits. ANCOVA and binary logistic regression were applied.

Results: Boys who spent less time in MVPA reported lower consumption of vegetables, fruits, cereals, yoghurt, milk, bread, pasta, candies and sugar-sweetened beverages (SSB) than boys who spent more time in MVPA $(P<0.05)$. Moreover, boys who spent less time in MVPA were more likely to consume fast foods and water than those in the highest MVPA tertile $(P<0.05)$. Girls who spent less time in MVPA reported lower consumption frequencies of vegetables, pasta, bread, yoghurt, candies, jam/honey and SSB than girls in the highest MVPA tertile $(P<0.05)$. Also, girls in the lowest MVPA tertile were more likely to consume fast foods and water than those with high levels of MVPA $(P<0 \cdot 05)$.

Conclusions: Food intake among European children varied with different levels of daily MVPA. Low time spent in MVPA was associated with lowest consumption of both high- and low-energy-dense foods and high fast-food consumption.
\end{abstract}

Keywords Physical activity Food intake Beverages intake Moderate-to-vigorous physical activity IDEFICS study
The worldwide prevalence of overweight and obesity combined rose by $27 \cdot 1 \%$ in adults and $47 \cdot 1 \%$ in children between 1980 and $2013^{(1,2)}$. However, in the last decade, overweight and obesity levels among children and adolescents have

$\dagger$ Alba M. Santaliestra Pasías and Jaime E. Llamas Dios contributed equally to this manuscript. shown a stabilization in several countries such as France ${ }^{(3)}$, the Netherlands ${ }^{(4)}$, Australia $^{(5)}$ and the USA ${ }^{(6)}$.

Low physical activity (PA) levels and unhealthy dietary habits are considered the most important risk factors for childhood obesity development ${ }^{(7)}$. In children and adolescents, high levels of PA are known to reduce cardiometabolic risk due to its effect on body fat ${ }^{(8)}$, lipid profile ${ }^{(9)}$ and blood 
pressure $^{(8)}$. Moreover, being physically active including three times per week of doing moderate-to-vigorous PA (MVPA) for at least $30 \mathrm{~min}$ was positively associated with a high cardiorespiratory fitness ${ }^{(10)}$, skeletal muscle $\operatorname{mass}^{(11)}$ and bone mineral density ${ }^{(8)}$.

In children and adolescents, the majority of studies have investigated the association between intake of fruits and vegetables and incidence of obesity ${ }^{(12)}$, type 2 diabetes ${ }^{(13)}$, $\mathrm{CHD}^{(14)}$ and some cancers ${ }^{(15)}$. The prevalence of European children involved in sports activities for more than $4 \mathrm{~h}$ /week varied between 1.1 and $4.4 \%$ for children younger than 6 years (girls and boys, respectively) and between 8.6 and $11.3 \%$ for children aged $6-9$ years (girls and boys, respectively) ${ }^{(16)}$. There are a few studies assessing the association between PA levels and food intakes in this age group ${ }^{(17-24)}$ and all these respective studies, however, used self-reported information.

In French and Saudi Arabian pre-adolescents, high PA levels measured with questionnaires were positively associated with high consumption of vegetables, fruits and fruit juices $^{(17,23)}$. However, no differences in intakes of sugarsweetened beverages (SSB) or French fries were observed according to PA level measured by a questionnaire among French adolescents ${ }^{(23)}$. In English adolescents, high sports participation was associated with high fresh food consumption $^{(18)}$, whereas in Iranian adolescents high PA levels (measured by metabolic equivalents of task (MET) derived from a validated PA questionnaire) were associated with high intakes of vegetables, fruits and dairy products ${ }^{(19)}$. In European adolescents, a positive association was observed between high PA levels obtained from the International Physical Activity Questionnaire and consumption of fruits and milk products ${ }^{(20)}$. Nevertheless, in the same sample of European adolescents, those males characterized by high MVPA levels had low-quality diets ${ }^{(22)}$. Also, Swiss adolescents practising sports nearly every day or every day had higher consumption frequencies for dairy products, fruit, fruit juices and salad than adolescents who did not practise sports regularly ${ }^{(24)}$. Moreover, a high proportion of girls who never or almost never practised sports had a lower consumption frequency of sports drinks and a higher consumption frequency of salted snacks than those who used to practise sports nearly every day or every day ${ }^{(24)}$.

To the authors' knowledge, the association between PA levels measured using accelerometers and food and beverage intakes in children across Europe has not been reported until now. The aim of the current study was to determine the association between MVPA levels and food and beverage intakes in European children aged 2-10 years.

\section{Materials and methods}

\section{Study population}

The IDEFICS (Identification and prevention of Dietary and lifestyle induced health EFfects In Children and infantS) project is a cohort study with an intervention on lifestyle and nutrition among children from eight European countries (Sweden, Germany, Hungary, Italy, Cyprus, Spain, Belgium and Estonia). Findings from the baseline survey of this multicentre cross-sectional study are the focus in the current analysis. Between September 2007 and June 2008, 16228 children aged 2-9 years from kindergartens, pre-schools and primary schools of selected regions fulfilled the study inclusion criteria (complete information on sex, height, weight and parental questionnaire).

\section{Sample for the current analysis}

For the current analysis, among the kindergarten, preschool or primary-school children from the eight IDEFCS participant countries with data available on sex, weight, height and parental questionnaire, only those children with complete information from the FFQ and valid accelerometer data for at least three days were included (7158 children, $49 \cdot 0 \%$ females).

Parents signed an informed consent and children were asked to give verbal assent before examination. Participants were free to refuse specific modules. For each country, participating centres obtained ethical approval from the local authorities as established in the Declaration of Helsinki.

\section{Socio-economic status}

The International Standard Classification of Education (ISCED) ${ }^{(25)}$ was used as proxy indicator of socio-economic status. In the current analysis, the highest parental educational level defined by the ISCED category was selected as the reference socio-economic status for the family. Parents self-reported their educational level in the core questionnaire. ISCED was registered from low to high educational level (1-6). The educational level was re-categorized into low (1-2), medium (3) and high (4-5).

\section{Anthropometric measurements}

Weight (in kilograms) and height (in centimetres) were measured by trained personnel according to a standardized protocol with participants barefoot and in underwear. Body weight was assessed in fasting status on a calibrated scale (Tanita electronic scale model BC 420 SMA with an adapter; Tanita Europe $\mathrm{GmbH}$ ) to the nearest $0.1 \mathrm{~kg}$. Height of the children was measured with a calibrated stadiometer (Seca telescopic height-measuring stadiometer model 225; Seca) to the nearest $0 \cdot 1 \mathrm{~cm}^{(26)}$. Finally, BMI was calculated as body weight in kilograms divided by the square of height in metres. BMI $Z$-scores and BMI categories were estimated according to Cole et $a l^{(27)}$.

\section{Physical activity}

PA was assessed objectively using a validated uniaxial accelerometer $^{(28)}$ (ActiGraph GT1M or Actitrainer; ActiGraph LLC). Children were instructed to wear the accelerometer for 
at least two weekdays and one weekend day. Parents were asked to complete a diary and to report all times when the accelerometer was not worn. The device was located on the right hip with an elastic belt. The children were asked to wear the device the whole day except during water-based activities and during sleep. The monitor was set to record PA in $15 \mathrm{~s}$ epochs. The accelerometer counts were categorized according to the cut-off points of Evenson to categorize different intensities of $\mathrm{PA}^{(29-31)}$ : sedentary, 0-100 counts per minute (cpm); light, 101-2295 cpm; moderate, 2296-4011 cpm; and vigorous, $\geq 4012 \mathrm{cpm}$. The selection of MVPA as main PA indicator was based on the PA guidelines established at $60 \mathrm{~min} / \mathrm{d}$ for children and adolescents ${ }^{(32,33)}$, and the Evenson cut-off points for MVPA levels were used to support comparability with other studies. Minutes were adjusted dividing the raw minutes by wearing time and multiplying the resulting fraction by the average wearing time. The time spent in MVPA was used to determine the association between PA and dietary intake. Consequently, time spent in MVPA was distributed in tertile groups separately by sex. Tertile 1 was named 'low MVPA', tertile 2 'medium MVPA' and tertile 3 'high MVPA.'

\section{$\boldsymbol{F F Q}$}

Food intake was assessed using the Children's Eating Habit Questionnaire (CEHQ) which includes a validated ${ }^{(34,35)}$ and reproducible $\mathrm{FFQ}^{(36)}$. Children's parents or guardians filled in the questionnaire at home by reporting the number of times the child consumed the specified food groups during a typical week in the previous month. The FFQ gives reproducible estimates of children's consumption frequencies derived from parental reports. Country-specific food examples were included to facilitate understanding. The CEHQFFQ contains forty-three food group items clustered into thirty-six according to their nutritional profiles. These food items were recoded into twenty-eight food groups according to their nutritional values: vegetables; potatoes; legumes; fruits; water; fruit juices; SSB; cereals; milk; yoghurt; fish; meat; eggs; soya replacement; cheese; chocolate; butter; honey; ketchup; bread; pasta; fast foods; nuts; savoury snacks; savoury pastries; candies; biscuits; and ice creams (see online supplementary material, Table S1, for foodstuffs included in each group). The twenty-eight food groups were used for the subsequent analysis.

Children's parents or guardians filled in the questionnaire at home by reporting the number of times the child consumed the specified food groups during a typical week in the previous month. The FFQ led participants to choose how many times they were consuming each kind of food using the options 'never/less than once a week', '1-3 times a week', '4-6 times a week', '1 time/day', '2 times/day', '3 times/day' and ' 4 or more times/day'. Food groups were recoded to establish groups organized by times daily: 'never/less than once a week' was recoded to 0 times/d, ' $1-3$ times a week' to $0 \cdot 28$ times/d and ' $4-6$ times a week' to $0 \cdot 71$ times/d. The options for
1 time/d, 2 times/d, 3 times/d and $\geq 4$ times/d were not modified. After that, food and beverage consumption were dichotomized by specific median consumption of each food or beverage group.

\section{Statistical analysis}

The statistical software package IBM SPSS Statistics for Windows version 22 was used for statistical analysis. Descriptive statistics, including means and SD, were calculated for each variable.

The $\chi^{2}$ test and the unpaired $t$ test were used to compare sample characteristics stratified by sex. All analyses were done separately for boys and girls, due to the interaction effect observed between sex and the independent variable. Differences in food intake per tertile of time spent in MVPA were analysed stratified by sex using ANCOVA, adjusted by age, socio-economic status and BMI. Binary logistic regression analyses were performed to obtain OR and $95 \% \mathrm{CI}$ for food and beverage intakes above the median by tertile of MVPA after adjusting for BMI $Z$-score, socio-economic status and country. $P$ values $<0.05$ were considered statistically significant.

\section{Results}

Table 1 shows descriptive information by sex regarding sociodemographic characteristics (age, socio-economic status, BMI), food and beverage consumption frequencies, and time spent doing PA. Sex differences were observed for age, food group consumption frequencies and PA levels $(P<0.05)$. Of the boys, $81.3 \%$, and of the girls, $79.0 \%$, were normal weight. Boys showed higher consumption frequencies of potatoes, meat, cereals, snacks, fruit juices, yoghurt, ketchup and biscuits $(P<0.05)$ and a lower consumption frequency of vegetables $(P<0.05)$ than girls. Total sedentary time was higher in girls compared with boys $(P<0.05)$, while boys accumulated more time in light PA and MVPA than girls $(P<0.05)$.

Table 2 shows the ANCOVA results (means and $\mathrm{SE}$ ) for food group consumption by tertile of MVPA for boys and girls. Boys who spent less time in MVPA (low MVPA) had lower consumption frequencies of vegetables, fruits, yoghurt and jam/honey, and higher consumption frequencies of cheese, pasta, biscuits and fast foods (pizza, hamburger and hot dog) than boys who were in the high MVPA group (Table 2). On the other hand, girls who spent less time in MVPA had lower consumption frequencies of vegetables, yoghurt, eggs, cereals, butter and SSB than girls who were in the high MVPA group. At the same time, these girls had higher consumption frequencies of water, cheese and fast foods than those who were in the high MVPA group (Table 2).

Table 3 shows the results of binary logistic regression analyses of the association between tertile of MVPA and food and beverage consumption, stratified by sex. 
Table 1 Descriptive characteristics of the European children from the IDEFICS study*

\begin{tabular}{|c|c|c|c|c|c|}
\hline & \multicolumn{2}{|c|}{ Boys ( $n$ 3618) } & \multicolumn{2}{|c|}{ Girls (n 3540) } & \multirow[b]{2}{*}{$P$ value } \\
\hline & Mean or $n$ & SE or $\%$ & Mean or $n$ & SE or $\%$ & \\
\hline $\begin{array}{l}\text { Age (years), mean and SE } \\
\text { Age category, } n \text { and } \%\end{array}$ & $6 \cdot 17$ & 0.03 & $6 \cdot 25$ & 0.03 & 0.023 \\
\hline $\begin{array}{l}2-5 \text { years } \\
6-9 \text { years }\end{array}$ & $\begin{array}{l}1111 \\
2507\end{array}$ & $\begin{array}{l}30 \cdot 7 \\
69 \cdot 3\end{array}$ & $\begin{array}{l}1033 \\
2507\end{array}$ & $\begin{array}{l}29 \cdot 2 \\
70 \cdot 8\end{array}$ & 0.083 \\
\hline \multicolumn{6}{|c|}{ Socio-economic status, $n$ and $\%$} \\
\hline $\begin{array}{l}\text { Low } \\
\text { Medium } \\
\text { High }\end{array}$ & $\begin{array}{r}305 \\
1349 \\
1964\end{array}$ & $\begin{array}{r}8 \cdot 4 \\
37 \cdot 3 \\
54 \cdot 3\end{array}$ & $\begin{array}{r}272 \\
1315 \\
1953\end{array}$ & $\begin{array}{r}7 \cdot 7 \\
37 \cdot 1 \\
55 \cdot 2\end{array}$ & 0.472 \\
\hline \multicolumn{5}{|l|}{ BMI category $\ddagger$, $n$ and \% } & 0.111 \\
\hline \multirow[t]{2}{*}{ Overweight or obese } & $\begin{array}{r}2943 \\
675\end{array}$ & $\begin{array}{l}81 \cdot 3 \\
18 \cdot 7\end{array}$ & $\begin{array}{r}2799 \\
741\end{array}$ & $\begin{array}{l}79.1 \\
21.9\end{array}$ & 0.008 \\
\hline & Mean & $95 \% \mathrm{Cl}$ & Mean & $95 \% \mathrm{Cl}$ & \\
\hline \multicolumn{6}{|c|}{ Food group consumption (times/d) } \\
\hline Vegetables & 1.17 & $1.14,1.21$ & 1.22 & $1.18,1.25$ & 0.042 \\
\hline Fruits & 1.41 & $1.36,1.46$ & 1.41 & $1.36,1.46$ & 0.449 \\
\hline Fruit juices & 1.19 & $1.13,1.24$ & 1.14 & $1.08,1.19$ & 0.031 \\
\hline Nuts & 0.13 & $0.12,0.014$ & 0.14 & $0.13,0.15$ & 0.063 \\
\hline Legumes & $0 \cdot 16$ & $0.14,0.19$ & $0 \cdot 15$ & $0.13,0.16$ & 0.272 \\
\hline Soya products & 0.02 & $0.01,0.03$ & 0.01 & $0.01,0.02$ & 0.062 \\
\hline Potatoes & 0.15 & $0.14,0.16$ & 0.13 & $0.13,0.14$ & 0.006 \\
\hline Bread & 1.57 & $1.53,1.62$ & 1.56 & $1.51,1.60$ & 0.276 \\
\hline Pasta & 0.49 & $0.47,0.51$ & 0.48 & $0.46,0.50$ & 0.514 \\
\hline Cereals & 0.61 & $0.59,0.64$ & 0.56 & $0.53,0.58$ & 0.000 \\
\hline Milk & 1.66 & $1.61,1.71$ & 1.61 & $1.56,1.67$ & $0 \cdot 157$ \\
\hline Yoghurt & 0.76 & $0.73,0.78$ & 0.73 & $0.70,0.76$ & 0.006 \\
\hline Cheese & 0.82 & $0.77,0.87$ & 0.85 & $0.77,0.93$ & 0.960 \\
\hline Butter & 0.86 & $0.83,0.90$ & 0.89 & $0.86,0.93$ & 0.436 \\
\hline Meat & 1.26 & $1.22,1.30$ & 1.18 & $1.14,1.21$ & 0.000 \\
\hline Fish & 0.30 & $0.29,0.32$ & 0.28 & $0.27,0.29$ & 0.067 \\
\hline Eggs & 0.34 & $0.33,0.36$ & 0.35 & $0.34,0.37$ & 0.571 \\
\hline Savoury pastries & 0.11 & $0.10,0.12$ & $0 \cdot 10$ & $0.09,0.11$ & 0.200 \\
\hline Biscuits & 0.34 & $0.32,0.35$ & 0.30 & $0.29,0.32$ & 0.003 \\
\hline Chocolate & 0.55 & $0.53,0.57$ & 0.53 & $0.51,0.55$ & 0.358 \\
\hline Candies & 0.29 & $0.27,0.30$ & 0.28 & $0.26,0.29$ & 0.978 \\
\hline Ice cream & 0.24 & $0.23,0.26$ & 0.24 & $0.23,0.26$ & 0.520 \\
\hline Jam and honey & 1.59 & $1.48,1.71$ & 1.47 & $1.37,1.58$ & 0.688 \\
\hline Fast foods & 0.34 & $0.33,0.36$ & 0.34 & $0.32,0.36$ & 0.123 \\
\hline Savoury snacks & 0.15 & $0.14,0.16$ & 0.13 & $0.12,0.14$ & 0.003 \\
\hline Ketchup & 0.24 & $0.23,0.25$ & 0.21 & $0.20,0.22$ & 0.001 \\
\hline Water & 4.50 & $4.40,4.59$ & 4.54 & $4.44,4.63$ & 0.423 \\
\hline \multirow[t]{2}{*}{ SSB } & 0.56 & $0.51,0.60$ & 0.52 & $0.47,0.57$ & 0.074 \\
\hline & Mean & SD & Mean & SD & \\
\hline \multicolumn{6}{|l|}{$\mathrm{PA}(\min / \mathrm{d})$} \\
\hline Sedentary time & 348.83 & $95 \cdot 22$ & 358.97 & 91.85 & 0.000 \\
\hline Light PA & 303.58 & $67 \cdot 18$ & $297 \cdot 39$ & 70.45 & 0.000 \\
\hline Moderate PA & 41.99 & $16 \cdot 37$ & 34.17 & 13.63 & 0.000 \\
\hline Vigorous PA & 13.73 & $9 \cdot 33$ & 11.76 & 8.03 & 0.000 \\
\hline Moderate-to-vigorous PA & 55.73 & 23.92 & 45.94 & 20.08 & 0.000 \\
\hline
\end{tabular}

IDEFICS, Identification and prevention of Dietary and lifestyle induced health EFfects In Children and infantS; SSB, sugar-sweetened beverages; PA, physical activity.

*The IDEFICS study was carried out between September 2007 and June 2008 in eight European countries (Sweden, Germany, Hungary, Italy, Cyprus, Spain, Belgium and Estonia).

†Gender differences using Pearson's $\chi^{2}$ test for categorical variables and the $t$ test for continuous variables. Results presented in bold font are statistically significant.

‡Based on Cole et al.'s cut-off values ${ }^{(27,45)}$.

Boys with low MVPA levels reported lower probability of consuming healthy foods such as vegetables $(\mathrm{OR}=0.75$, $95 \%$ CI $0 \cdot 63,0 \cdot 89)$, cereals $(\mathrm{OR}=0.62,95 \%$ CI $0.51,0 \cdot 76)$ and milk (OR $=0.83,95 \%$ CI $0.70,0.99)$ than boys in the high MVPA group. Also, boys from the low and medium MVPA groups reported lower probability of consuming unhealthy beverages such as SSB (i.e. sweetened and diet drinks; $\mathrm{OR}=0.81,95 \%$ CI $0.68,0.96$ and $\mathrm{OR}=0.79,95 \%$ CI $0.67,0.93$, respectively) than those who were in the high MVPA group.

Boys in the low and medium MVPA groups showed lower probability of consuming several healthy groups 
Table 2 ANCOVA of food group consumption (times/d), by tertile of MVPA level and by sex ${ }^{*}$ among the European children from the IDEFICS study†

\begin{tabular}{|c|c|c|c|c|c|c|c|c|c|c|c|c|}
\hline \multirow[b]{3}{*}{ Food consumption (times/d) } & \multicolumn{6}{|c|}{ Boys } & \multicolumn{6}{|c|}{ Girls } \\
\hline & \multicolumn{2}{|c|}{ Low MVPA } & \multicolumn{2}{|c|}{ Medium MVPA } & \multicolumn{2}{|c|}{ High MVPA } & \multicolumn{2}{|c|}{ Low MVPA } & \multicolumn{2}{|c|}{ Medium MVPA } & \multicolumn{2}{|c|}{ High MVPA } \\
\hline & Mean & SE & Mean & SE & Mean & SE & Mean & SE & Mean & SE & Mean & SE \\
\hline Vegetables & $1 \cdot 10^{b}$ & 0.03 & $1 \cdot 16$ & 0.03 & $1.25^{\mathrm{b}}$ & 0.03 & $1.09^{a, b}$ & 0.03 & $1.25^{a}$ & 0.03 & $1.32^{b}$ & 0.03 \\
\hline Fruits & $1.35^{\mathrm{b}}$ & 0.04 & 1.37 & 0.04 & $1.53^{b}$ & 0.04 & 1.36 & 0.04 & 1.45 & 0.04 & 1.43 & 0.04 \\
\hline Fruit juice & 1.19 & 0.05 & 1.17 & 0.05 & $1 \cdot 19$ & 0.05 & $1 \cdot 12$ & 0.05 & 1.14 & 0.05 & $1 \cdot 15$ & 0.05 \\
\hline Nuts & 0.12 & 0.01 & 0.14 & 0.01 & 0.13 & 0.01 & 0.13 & 0.01 & 0.15 & 0.01 & 0.15 & 0.01 \\
\hline Legumes & 0.16 & 0.03 & 0.19 & 0.02 & 0.16 & 0.02 & 0.13 & 0.02 & 0.14 & 0.01 & 0.16 & 0.01 \\
\hline Soya products & 0.02 & 0.01 & 0.03 & 0.01 & 0.02 & 0.01 & 0.02 & 0.01 & 0.02 & 0.01 & 0.02 & 0.01 \\
\hline Potatoes & 0.16 & 0.01 & 0.15 & 0.01 & 0.14 & 0.01 & 0.14 & 0.01 & 0.14 & 0.01 & 0.13 & 0.01 \\
\hline Bread & 1.52 & 0.03 & 1.53 & 0.03 & 1.54 & 0.03 & 1.53 & 0.03 & 1.51 & 0.03 & 1.53 & 0.03 \\
\hline Pasta & $0.53^{\mathrm{a}, \mathrm{b}}$ & 0.01 & $0.47^{\mathrm{a}}$ & 0.01 & $0.48^{b}$ & 0.01 & $0.52^{\mathrm{a}}$ & 0.02 & $0.45^{a}$ & 0.01 & 0.48 & 0.02 \\
\hline Cereals & 0.60 & 0.02 & 0.61 & 0.02 & 0.63 & 0.02 & $0.51^{b}$ & 0.02 & 0.56 & 0.02 & $0.60^{\mathrm{b}}$ & 0.02 \\
\hline Milk & 1.58 & 0.05 & 1.69 & 0.05 & 1.70 & 0.05 & 1.54 & 0.05 & 1.65 & 0.05 & 1.62 & 0.05 \\
\hline Yoghurt & $0.66^{\mathrm{a}, \mathrm{b}}$ & 0.02 & $0.79^{a}$ & 0.02 & $0.82^{b}$ & 0.02 & $0.65^{\mathrm{a}, \mathrm{b}}$ & 0.02 & $0.76^{a}$ & 0.02 & $0.78^{b}$ & 0.02 \\
\hline Cheese & $0.97^{a, b}$ & 0.04 & $0.72^{\mathrm{a}}$ & 0.05 & $0.73^{\mathrm{b}}$ & 0.05 & $1.00^{a, b}$ & 0.06 & $0.75^{a}$ & 0.07 & $0.69^{b}$ & 0.08 \\
\hline Butter & 0.53 & 0.02 & 0.56 & 0.02 & 0.60 & 0.02 & $0.51^{a, b}$ & 0.03 & $0.62^{a}$ & 0.02 & $0.65^{b}$ & 0.04 \\
\hline Meat & 1.21 & 0.04 & 1.27 & 0.04 & 1.30 & 0.03 & $1 \cdot 13$ & 0.03 & $1 \cdot 18$ & 0.03 & 1.23 & 0.03 \\
\hline Fish & 0.33 & 0.01 & 0.29 & 0.01 & 0.30 & 0.01 & 0.29 & 0.01 & 0.28 & 0.01 & 0.29 & 0.01 \\
\hline Eggs & 0.33 & 0.02 & 0.36 & 0.02 & 0.35 & 0.02 & $0.33^{b}$ & 0.01 & 0.35 & 0.01 & $0.38^{b}$ & 0.01 \\
\hline Savoury pastries & 0.12 & 0.01 & 0.11 & 0.01 & 0.10 & 0.01 & 0.11 & 0.01 & 0.11 & 0.01 & 0.09 & 0.01 \\
\hline Biscuits & $0.37^{\mathrm{b}}$ & 0.01 & 0.34 & 0.01 & $0.31^{b}$ & 0.01 & 0.32 & 0.01 & 0.31 & 0.01 & 0.29 & 0.01 \\
\hline Chocolate & 0.57 & 0.02 & 0.53 & 0.02 & 0.53 & 0.02 & 0.55 & 0.02 & 0.54 & 0.02 & 0.52 & 0.02 \\
\hline Candies & 0.29 & 0.02 & 0.27 & 0.02 & 0.32 & 0.01 & 0.27 & 0.01 & 0.27 & 0.01 & 0.30 & 0.02 \\
\hline Ice cream & 0.27 & 0.01 & 0.24 & 0.01 & 0.24 & 0.01 & 0.26 & 0.01 & 0.23 & 0.01 & 0.25 & 0.01 \\
\hline Jam and honey & $0.23^{b}$ & 0.01 & 0.24 & 0.01 & $0.28^{\mathrm{b}}$ & 0.01 & 0.25 & 0.01 & 0.24 & 0.01 & 0.27 & 0.01 \\
\hline Fast foods & $0.38^{\mathrm{b}}$ & 0.02 & $0.36^{c}$ & 0.02 & $0.30^{\mathrm{b}, \mathrm{c}}$ & 0.02 & $0.36^{b}$ & 0.01 & $0.37^{\mathrm{c}}$ & 0.01 & $0.30^{\mathrm{b}, \mathrm{c}}$ & 0.02 \\
\hline Savoury snacks & 0.15 & 0.01 & 0.15 & 0.01 & 0.15 & 0.01 & 0.14 & 0.01 & 0.13 & 0.01 & 0.13 & 0.01 \\
\hline Ketchup & $0.22^{\mathrm{a}}$ & 0.01 & $0.26^{a}$ & 0.01 & 0.24 & 0.01 & 0.20 & 0.01 & 0.22 & 0.01 & 0.22 & 0.01 \\
\hline Water & 4.63 & 0.08 & 4.49 & 0.08 & 4.41 & 0.08 & $4 \cdot 76^{a, b}$ & 0.08 & $4.47^{\mathrm{a}}$ & 0.08 & $4.42^{\mathrm{b}}$ & 0.08 \\
\hline SSB & 0.58 & 0.04 & 0.50 & 0.04 & 0.60 & 0.04 & $0.45^{\mathrm{b}}$ & 0.04 & $0.45^{\mathrm{c}}$ & 0.04 & $0.66^{\mathrm{b}, \mathrm{c}}$ & 0.04 \\
\hline
\end{tabular}

MVPA, moderate-to-vigorous physical activity; IDEFICS, Identification and prevention of Dietary and lifestyle induced health EFfects In Children and infantS; SSB, sugar-sweetened beverages.

${ }_{a, b, c}$ For boys and girls separately, mean values were significantly different between ${ }^{\text {afirst }}$ and second tertile, ${ }^{b}$ first and third tertile and ${ }^{c}$ second and third tertile of MVPA $(P<0.05)$

${ }^{*}$ Covariates were age, BMI and socio-economic status. Results presented in bold font are statistically significant.

†The IDEFICS study was carried out between September 2007 and June 2008 in eight European countries (Sweden, Germany, Hungary, Italy, Cyprus, Spain, Belgium and Estonia).

such as fruits $(\mathrm{OR}=0.75,95 \% \mathrm{CI} 0.62,0.89$ and $\mathrm{OR}=0.83$, $95 \%$ CI $0.70, \quad 0.99$, respectively) and yoghurt $(\mathrm{OR}=0 \cdot 73,95 \% \mathrm{CI} 0 \cdot 61,0 \cdot 87$ and $\mathrm{OR}=0 \cdot 81,95 \% \mathrm{CI} 0 \cdot 68$, $0 \cdot 97$, respectively) than boys in the high MVPA group. On the other hand, boys from low and medium MVPA groups showed higher probability of consuming unhealthy foods such as fast foods (i.e. pizza main dish and hamburger, hot dog, kebab, wrap and falafel; OR =1.31, $95 \%$ CI 1.10, 1.55 and $\mathrm{OR}=1 \cdot 22,95 \%$ CI $1 \cdot 03,1 \cdot 43$, respectively) than boys with high MVPA levels.

Girls with low MVPA reported lower probability of consuming healthy foods such as vegetables $(\mathrm{OR}=0.85$, $95 \%$ CI $0.48,0 \cdot 69)$, cereals ( $\mathrm{OR}=0.65,95 \%$ CI $0.53,0.79)$ and yoghurt ( $\mathrm{OR}=0 \cdot 72,95 \% \mathrm{CI} 0 \cdot 60,0 \cdot 86)$ than girls in the high MVPA group.

In addition, girls from low and medium MVPA groups showed lower probability of consuming unhealthy foods such as SSB (OR =0.68, 95\% CI 0.57, 0.81 and OR =0.80, $95 \%$ CI $0.67,0.94$, respectively) and candies (OR $=0.55$, $95 \%$ CI $0.46,0.66$ and $\mathrm{OR}=0.80,95 \%$ CI $0.68,0.95$, respectively) than girls in the high MVPA group. On the other hand, girls from low and medium MVPA groups showed higher probability of consuming unhealthy foods such as fast foods $(\mathrm{OR}=1.35,95 \% \mathrm{CI} 1 \cdot 14,1.61$ and $\mathrm{OR}=1 \cdot 36,95 \% \mathrm{CI} 1 \cdot 15,1 \cdot 61$, respectively) than girls in the high MVPA group.

\section{Discussion}

To our knowledge, the present study is one of the first performed in children across Europe aged 2-10 years that analyses the relationship between consumption of several food groups and levels of MVPA. The current results show that children who spent more time in MVPA are not necessarily consumers of healthy foods as expected. Also, different food intakes varied between levels of MVPA in European children. In our sample, only $30 \%$ of the sample met the current PA recommendations taking into consideration the Evenson cut-off points for MVPA.

A recent review suggested that few studies have included objective measures such as accelerometry and during school play periods to reliable assess trends in PA, 
Table 3 Binary logistic regression analysis predicting food group consumption above the median, by tertile of MVPA level and by sex*, among the European children from the IDEFICS study†

\begin{tabular}{|c|c|c|c|c|c|c|c|c|c|c|}
\hline \multirow[b]{3}{*}{ Food consumption } & \multicolumn{5}{|c|}{ Boys } & \multicolumn{5}{|c|}{ Girls } \\
\hline & \multicolumn{2}{|c|}{ Low MVPA } & \multicolumn{2}{|c|}{ Medium MVPA } & \multirow{2}{*}{$\frac{\text { High MVPA }}{\text { OR }}$} & \multicolumn{2}{|c|}{ Low MVPA } & \multicolumn{2}{|c|}{ Medium MVPA } & \multirow{2}{*}{$\frac{\text { High MVPA }}{\text { OR }}$} \\
\hline & OR & $95 \% \mathrm{Cl}$ & OR & $95 \% \mathrm{Cl}$ & & OR & $95 \% \mathrm{Cl}$ & OR & $95 \% \mathrm{Cl}$ & \\
\hline Vegetables & 0.75 & $0.63,0.89$ & 0.85 & $0.72,1.01$ & 1.00 (ref.) & 0.85 & $0.48,0.69$ & 0.89 & $0.45,1.06$ & 1.00 (ref.) \\
\hline Fruits & 0.75 & $0.62,0.89$ & 0.83 & $0.70,0.99$ & 1.00 (ref.) & 0.92 & $0.77,1.11$ & 1.13 & $0.95,1.35$ & 1.00 (ref.) \\
\hline Fruit juices & 0.95 & $0.80,1.12$ & 1.07 & $0.91,1.27$ & 1.00 (ref.) & 0.99 & $0.84,1.18$ & 1.03 & $0.87,1.22$ & 1.00 (ref.) \\
\hline Nuts & 0.87 & $0.73,1.04$ & 1.05 & $0.89,1.25$ & 1.00 (ref.) & 0.90 & $0.76,1.08$ & $1 \cdot 12$ & $0.95,1.33$ & 1.00 (ref.) \\
\hline Legumes & 0.70 & $0.46,1.07$ & 0.87 & $0.54,1.41$ & 1.00 (ref.) & 0.92 & $0.61,1.38$ & 0.88 & $0.53,1.45$ & 1.00 (ref.) \\
\hline Soya products & 0.82 & $0.51,1.31$ & 0.90 & $0.57,1.41$ & 1.00 (ref.) & 0.72 & $0.43,1.18$ & 0.94 & $0.60,1.48$ & 1.00 (ref.) \\
\hline Potatoes & 0.96 & $0.81,1.13$ & 1.04 & $0.88,1.22$ & 1.00 (ref.) & 1.07 & $0.90,1.26$ & 1.22 & $0.03,1.44$ & 1.00 (ref.) \\
\hline Bread & 0.84 & $0.71,0.99$ & 0.87 & $0.74,1.03$ & 1.00 (ref.) & 0.98 & $0.83,1.16$ & 1.09 & $0.92,1.29$ & 1.00 (ref.) \\
\hline Pasta & 0.55 & $0.40,0.76$ & 0.66 & $0.47,0.91$ & 1.00 (ref.) & 0.71 & $0.52,0.97$ & 0.82 & $0.60,1.12$ & 1.00 (ref.) \\
\hline Cereals & 0.62 & $0.51,0.76$ & 0.80 & $0.66,0.98$ & 1.00 (ref.) & 0.65 & $0.53,0.79$ & 0.86 & $0.71,1.05$ & 1.00 (ref.) \\
\hline Milk & 0.83 & $0.70,0.99$ & 0.92 & $0.78,1.09$ & 1.00 (ref.) & 0.94 & $0.79,1.11$ & 1.07 & $0.91,1.27$ & 1.00 (ref.) \\
\hline Yoghurt & 0.73 & $0.61,0.87$ & 0.81 & $0.68,0.97$ & 1.00 (ref.) & 0.72 & $0.60,0.86$ & 0.96 & $0.81,1.15$ & 1.00 (ref.) \\
\hline Cheese & $1 \cdot 10$ & $0.77,1.56$ & 0.98 & $0.71,1.37$ & 1.00 (ref.) & 0.85 & $0.58,1.25$ & 0.90 & $0.63,1.30$ & 1.00 (ref.) \\
\hline Butter & 0.82 & $0.69,0.97$ & 0.91 & $0.77,1.08$ & 1.00 (ref.) & 0.54 & $0.45,0.64$ & 0.90 & $0.75,1.07$ & 1.00 (ref.) \\
\hline Meat & 0.90 & $0.74,1.11$ & 0.93 & $0.77,1.13$ & 1.00 (ref.) & 0.83 & $0.68,1.02$ & 0.94 & $0.77,1.14$ & 1.00 (ref.) \\
\hline Fish & 0.97 & $0.80,1.16$ & 0.94 & $0.79,1.13$ & 1.00 (ref.) & 0.97 & $0.81,1.17$ & 0.97 & $0.81,1.17$ & 1.00 (ref.) \\
\hline Eggs & 1.02 & $0.85,1.23$ & 1.04 & $0.86,1.24$ & 1.00 (ref.) & 0.88 & $0.73,1.07$ & 0.99 & $0.82,1.20$ & 1.00 (ref.) \\
\hline Savoury pastries & 1.03 & $0.86,1.23$ & 1.17 & $0.98,1.39$ & 1.00 (ref.) & 1.11 & $0.82,1.34$ & 1.27 & $1.06,1.52$ & 1.00 (ref.) \\
\hline Biscuits & 0.81 & $0.68,0.97$ & 0.91 & $0.77,1.08$ & 1.00 (ref.) & 0.64 & $0.54,0.77$ & 0.91 & $0.76,1.09$ & 1.00 (ref.) \\
\hline Chocolate & 0.94 & $0.77,1.16$ & 0.93 & $0.76,1.13$ & 1.00 (ref.) & 0.96 & $0.78,1.17$ & 1.09 & $0.89,1.33$ & 1.00 (ref.) \\
\hline Candies & 0.54 & $0.45,0.64$ & 0.73 & $0.62,0.86$ & 1.00 (ref.) & 0.55 & $0.46,0.66$ & 0.80 & $0.68,0.95$ & 1.00 (ref.) \\
\hline Ice cream & 0.86 & $0.73,1.02$ & 0.89 & $0.75,1.05$ & 1.00 (ref.) & 0.94 & $0.80,1.12$ & 0.98 & $0.83,1.16$ & 1.00 (ref.) \\
\hline Jam and honey & 0.75 & $0.63,0.88$ & 0.93 & $0.79,1 \cdot 10$ & 1.00 (ref.) & 0.78 & $0.66,0.92$ & 0.87 & $0.74,1.03$ & 1.00 (ref.) \\
\hline Fast foods & 1.31 & $1.10,1.55$ & 1.22 & $1.03,1.43$ & 1.00 (ref.) & 1.35 & $1.14,1.61$ & 1.36 & $1.15,1.61$ & 1.00 (ref.) \\
\hline Savoury snacks & 0.86 & $0.72,1.01$ & 0.98 & $0.83,1.15$ & 1.00 (ref.) & 0.99 & $0.83,1.18$ & 1.00 & $0.85,1.18$ & 1.00 (ref.) \\
\hline Ketchup & 0.64 & $0.54,0.76$ & 0.83 & $0.71,0.98$ & 1.00 (ref.) & 0.70 & $0.59,0.83$ & 0.95 & $0.80,1.11$ & 1.00 (ref.) \\
\hline Water & 1.43 & $1.20,1.69$ & $1 \cdot 10$ & $0.93,1.30$ & 1.00 (ref.) & 1.38 & $1.16,1.64$ & 1.06 & $0.90,1.26$ & 1.00 (ref.) \\
\hline SSB & 0.81 & $0.68,0.96$ & 0.79 & $0.67,0.93$ & 1.00 (ref.) & 0.68 & $0.57,0.81$ & 0.80 & $0.67,0.94$ & 1.00 (ref.) \\
\hline
\end{tabular}

MVPA, moderate-to-vigorous physical activity; IDEFICS, Identification and prevention of Dietary and lifestyle induced health EFfects In Children and infantS; SSB, sugar-sweetened beverages; ref., reference category.

${ }^{*}$ Covariates were BMI Z-score, socio-economic status and country. Results presented in bold font are statistically significant.

†The IDEFICS study was carried out between September 2007 and June 2008 in eight European countries (Sweden, Germany, Hungary, Italy, Cyprus, Spain, Belgium and Estonia).

highlighting a need for further research $^{(37)}$. Previous studies have shown that a low proportion of IDEFICS participants achieved international recommendations for PA levels ${ }^{(16,38)}$ of $60 \mathrm{~min} / \mathrm{d}^{(33)}$. Also in the current investigation, the studied groups did not spend enough daily time in MVPA to achieve the current recommendations. As the proportion of children complying with the recommendations was generally low, we decided to categorize the sample by tertiles of MVPA. The decision to set the lowest tertile (tertile 1) of MVPA duration as reference was based on the fact that this group of children is exposed to high risk of developing several diseases because they do not meet the current PA recommendations. We observed that more MVPA was practised by boys, and higher daily MVPA was associated with food and beverage consumption.

Some studies have shown a positive association among high levels of PA and vegetable and fruit intake (19,20,39,40). $^{(19)}$. Our results are in line with Kelishadi et al. ${ }^{(19)}$ who reported a positive association between PA level and vegetable intake in Iranian boys and girls, and with fruit consumption only in boys.

Results of the Healthy Lifestyle in Europe by Nutrition in Adolescence (HELENA) study showed a negative association between bread and cereal consumption and PA level derived from a PA questionnaire in European female adolescents ${ }^{(20)}$. In contrast, our results have shown a positive association between the probability of cereal consumption and MVPA in European boys and girls. Ottevaere et $a l .{ }^{(20)}$ reported a positive association between consumption of grain products and PA level in European boys. In both sexes, our results have shown a positive association between the probability of pasta consumption and time spent in MVPA.

Moreover, in the HELENA study population, high consumption of milk and cheese was also observed in the most active girls ${ }^{(20)}$. Our results have shown a positive association between MVPA level and the probability of milk consumption in both sexes and a negative association between MVPA level and cheese consumption. Additionally, Ottevaere et al. ${ }^{(20)}$ showed a positive association between consumption of water, meat and meat products and PA in European boys. Our results are in concordance with those obtained by that group, being statistically significant in both sexes. In a sample of Pakistani children $^{(39)}$, no association was found between PA level derived from a questionnaire and fast-food consumption. 
However, our results have shown a higher probability of consumption of fast-food products in those children with low MVPA levels compared with those from the high MVPA group. Furthermore, in the HELENA study no association was found between snack or ketchup consumption and PA level ${ }^{(20)}$; but in our European children a higher probability of ketchup consumption was observed in those with high MVPA levels. Our results are in line with those of Kelishadi et $a l .{ }^{(19)}$ who showed a positive association between candy consumption and PA level derived from a questionnaire validated by accelerometer in Islamic students. On the other hand, Ranjit et $a l .{ }^{(40)}$ did not report any relationship between PA level obtained from a questionnaire and SSB consumption in American children. In the IDEFICS study, girls showed a positive association between MVPA and the probability of SSB intake.

The differences between studies could be caused by differences between the countries of origin or sample size. On the other hand, our results were focused only on MVPA levels, while other studies included total PA. Moreover, the use of different measurements (i.e. $\mathrm{min} / \mathrm{d}$, times/week, sports participation, in leisure time, etc.) to estimate the PA levels could have influenced the observed results. Along the same line, the comparability between studies is hampered because different measures are used to assess PA levels. Also, some of them were self-reported, whereas accelerometery gave the opportunity to focus on more reliable PA values in the current study.

\section{Strengths and limitations}

Several strengths can be noted in the present study. First, it is important to consider the broad range of examinations performed at European level, including a large number of participants from eight countries. In addition, the IDEFICS study was developed using a standardized and harmonized protocol across all involved countries. ActiGraph was the model which provided the strongest validity in the IDEFICS validation study ${ }^{(41)}$. Nevertheless, the limitation that the fixed $60 \mathrm{~s}$ epoch underestimated MVPA should be taken into consideration ${ }^{(42)}$. Also, the present study had additional limitations. It was an explorative investigation using cross-sectional data and no causal conclusions can be drawn. Moreover, although the FFQ is a useful tool to assess qualitative food intake and it has been validated for this population group ${ }^{(36)}$, it is necessary to take into consideration that the FFQ was parental-reported. The assessment of energy balance-related behaviours has been shown to be difficult and complex in young children ${ }^{(43)}$, and self- or parental reports are a source of error because they depend on participant's memory and these estimates may be influenced by the desire to report healthy habits. Also, parents do not always know what their children are eating. We therefore acknowledge that a reporting bias cannot be excluded entirely. We also should mention that the data assessed were collected 10 years ago; however, there is limited research on temporal trends in children's $\mathrm{PA}^{(37)}$. The current evidence suggests that few changes have occurred in the last 20 years and that organized sport trends are inconsistent between countries ${ }^{(37)}$.

\section{Conclusions}

Considering the results obtained from the IDEFICS study, we observed a low frequency of consuming fruits and vegetables in children that makes it difficult to for them reach the current intake recommendations. Moreover, the children who participated in the study had a high consumption frequency of sweets and candies, according to recommendations for food consumption ${ }^{(44)}$.

Current results showed that dietary intake was different between children with different levels of MVPA. Moreover, our results showed that children who spent more time in MVPA did not necessarily consume healthy foods as expected. Food intakes varied between levels of MVPA in European children. In both sexes, low time spent in MVPA was associated with high consumption of high-energy-dense foods such as cheese or fast foods; and in boys with low consumption of low-energy-dense foods as vegetables, fruits or yoghurts. Moreover, in girls, low time spent in MVPA was associated with low consumption of low-energy-dense foods like milk, eggs, pasta and cereals, and low consumption of high-energy-dense foods such as yoghurt and nuts. Furthermore, it is important to perform additional research in order to know the relationship between diet and MVPA.

\section{Acknowledgements}

Acknowledgements: The authors thank all members of the study teams and especially the children and their parents for their participation in the study. This work was carried out as a part of the IDEFICS Study and is published on behalf of its European Consortium (http://www.idefics. eu). Financial support: The authors gratefully acknowledge the financial support of the European Community within the Sixth RTD Framework Programme, Contract No. 016181 (FOOD); as well as the Ministry of Science and Innovation (MICINN) and the European Region Development Fund (Fondo Europeo de Desarrollo Regional, FEDER) for their financial support. The funders had no role in the design, analysis or writing of this article. Disclaimer: The information in this document reflects the authors' view and is provided as is. No guarantee or warranty is given that the information is fit for any particular purpose. The reader, therefore, uses the information at his/her sole risk and liability. Conflict of interest: The authors declare no conflict of interest. Authorship: A.M.S.-P. and J.E.L.D. had full access to all the data in the study and take responsibility for the integrity of the data and the accuracy of the data analysis. Study concept and design: A.M.S.-P., J.E.L.D., S.D.H., G.E., M.T., T.V., V.P. and L.A.M. 
Acquisition of data: A.M.S.-P., A.H., R.F., F.L, T.V., D.M. and L.A.M. Analysis and interpretation of data: A.M.S.-P., J.E.L.D., O.S., A.H., S.D.H., G.E., R.F., F.L., M.T., T.V., V.P. and L.A.M. Critical revision of the manuscript for important intellectual content: A.M.S.-P., J.E.L.D., O.S., A.H., S.D.H., G.E., R.F., F.L., M.T., T.V., V.P. and L.A.M. Statistical analysis: A.M.S.-P. J.E.L.D. and L.A.M. Administrative, technical and material support: A.M.S.-P., O.S., A.H., M.T., T.V., V.P. and L.A.M. Study supervision: S.D.H., G.E., M.T., T.V., V.P. and L.A.M. Ethics of human subject participation: For each country, participating centres obtained ethical approval from the local authorities as established in the Declaration of Helsinki. Parents signed an informed consent and children were asked to give verbal assent before examination. Specific ethics committee of each city involved: Ethics Committee, University Hospital, Ghent (Belgium); Cyprus National Bioethics Committee (Cyprus); Tallinn Medical Research Ethics Committee (Estonia); Ethics Committee, University of Bremen (Germany); Egészségügyi Tudományos Tanács, Pécs (Hungary); Comitato Etico, ASL Avellino (Italy); Comité Ético de Investigación, Clínica de Aragón (CEICA) (Spain); Regional Ethics Review Board, University of Gothenburg (Sweden).

\section{Supplementary material}

To view supplementary material for this article, please visit https://doi.org/10.1017/S1368980018000046

\section{References}

1. de Onis M, Blossner M \& Borghi E (2010) Global prevalence and trends of overweight and obesity among preschool children. Am J Clin Nutr 92, 1257-1264.

2. Ng M, Fleming T, Robinson M et al. (2014) Global, regional, and national prevalence of overweight and obesity in children and adults during 1980-2013: a systematic analysis for the Global Burden of Disease Study 2013. Lancet 384, 766-781.

3. Lioret S, Touvier M, Dubuisson C et al. (2009) Trends in child overweight rates and energy intake in France from 1999 to 2007: relationships with socioeconomic status. Obesity (Silver Spring) 17, 1092-1100.

4. de Wilde JA, van Dommelen P, Middelkoop BJ et al. (2009) Trends in overweight and obesity prevalence in Dutch, Turkish, Moroccan and Surinamese South Asian children in the Netherlands. Arch Dis Cbild 94, 795-800.

5. Olds TS, Tomkinson GR, Ferrar KE et al. (2010) Trends in the prevalence of childhood overweight and obesity in Australia between 1985 and 2008. Int J Obes (Lond) 34, 57-66.

6. Ogden CL, Carroll MD \& Flegal KM (2008) High body mass index for age among US children and adolescents, 2003-2006. JAMA 299, 2401-2405.

7. Herman KM, Chaput JP, Sabiston CM et al. (2015) Combined physical activity/sedentary behaviour associations with indices of adiposity in 8- to 10-year-old children. J Phys Act Health 12, 20-29.

8. Janssen I \& Leblanc AG (2010) Systematic review of the health benefits of physical activity and fitness in schoolaged children and youth. Int J Behav Nutr Phys Act 7, 40.
9. Ara I, Vicente-Rodriguez G, Jimenez-Ramirez J et al. (2004) Regular participation in sports is associated with enhanced physical fitness and lower fat mass in prepubertal boys. Int J Obes Relat Metab Disord 28, $1585-1593$.

10. Hsieh PL, Chen ML, Huang CM et al. (2014) Physical activity, body mass index, and cardiorespiratory fitness among school children in Taiwan: a cross-sectional study. Int J Environ Res Public Health 11, 7275-7285.

11. Detter F, Nilsson JA, Karlsson C et al. (2014) A 3-year schoolbased exercise intervention improves muscle strength - a prospective controlled population-based study in 223 children. BMC Musculoskelet Disord 15, 353.

12. Vilchis-Gil J, Galvan-Portillo M, Klunder-Klunder $\mathrm{M}$ et al. (2015) Food habits, physical activities and sedentary lifestyles of eutrophic and obese school children: a case-control study. BMC Public Health 15, 124.

13. Li M, Fan Y, Zhang X et al. (2014) Fruit and vegetable intake and risk of type 2 diabetes mellitus: meta-analysis of prospective cohort studies. BMJ Open 4, e005497.

14. He FJ, Nowson CA, Lucas M et al. (2007) Increased consumption of fruit and vegetables is related to a reduced risk of coronary heart disease: meta-analysis of cohort studies. J Hum Hypertens 21, 717-728.

15. Holman DM \& White MC (2011) Dietary behaviors related to cancer prevention among pre-adolescents and adolescents: the gap between recommendations and reality. Nutr J 10, 60 .

16. Santaliestra-Pasias AM, Mouratidou $\mathrm{T}$, Verbestel $\mathrm{V}$ et al. (2014) Physical activity and sedentary behaviour in European children: the IDEFICS study. Public Health Nutr 17, 2295-2306.

17. Al-Hazzaa HM, Abahussain NA, Al-Sobayel HI et al. (2011) Physical activity, sedentary behaviors and dietary habits among Saudi adolescents relative to age, gender and region. Int J Behav Nutr Phys Act 8, 140.

18. Coulson NS, Eiser C \& Eiser JR (1997) Diet, smoking and exercise: interrelationships between adolescent health behaviours. Child Care Health Dev 23, 207-216.

19. Kelishadi R, Ardalan G, Gheiratmand R et al. (2007) Association of physical activity and dietary behaviours in relation to the body mass index in a national sample of Iranian children and adolescents: CASPIAN Study. Bull World Health Organ 85, 19-26.

20. Ottevaere C, Huybrechts I, Beghin L et al. (2011) Relationship between self-reported dietary intake and physical activity levels among adolescents: the HELENA study. Int $J$ Behav Nutr Phys Act 8, 8.

21. Storey KE, Forbes LE, Fraser SN et al. (2009) Diet quality, nutrition and physical activity among adolescents: the Web-SPAN (Web-Survey of Physical Activity and Nutrition) project. Public Health Nutr 12, 2009-2017.

22. Ottevaere C, Huybrechts I, Benser J et al. (2011) Clustering patterns of physical activity, sedentary and dietary behavior among European adolescents: the HELENA study. BMC Public Health 11, 328.

23. Platat C, Perrin AE, Oujaa M et al. (2006) Diet and physical activity profiles in French preadolescents. Br J Nutr 96, 501-507.

24. Cavadini C, Decarli B, Grin J et al. (2000) Food habits and sport activity during adolescence: differences between athletic and non-athletic teenagers in Switzerland. Eur J Clin Nutr 54, Suppl. 1, S16-S20.

25. United Nations Educational, Scientific and Cultural Organization (1997) International Standard Classification of Education. Geneva: UNESCO.

26. Nagy P, Kovacs E, Moreno LA et al. (2016) Percentile reference values for anthropometric body composition indices in European children from the IDEFICS study. Int $J$ Obes (Lond) 38, Suppl. 2, S15-S25. 
27. Cole TJ, Bellizzi MC, Flegal KM et al. (2000) Establishing a standard definition for child overweight and obesity worldwide: international survey. BMJ 320, 1240-1243.

28. Konstabel K, Veidebaum T, Verbestel V et al. (2014) Objectively measured physical activity in European children: the IDEFICS study. Int J Obes (Lond) 38, Suppl. 2, S135-S143.

29. Evenson KR, Catellier DJ, Gill K et al. (2008) Calibration of two objective measures of physical activity for children. J Sports Sci 26, 1557-1565.

30. Janssen X, Cliff DP, Reilly JJ et al. (2013) Predictive validity and classification accuracy of ActiGraph energy expenditure equations and cut-points in young children. PLoS One $\mathbf{8}$, e79124.

31. Trost SG, Loprinzi PD, Moore R et al. (2011) Comparison of accelerometer cut points for predicting activity intensity in youth. Med Sci Sports Exerc 43, 1360-1368.

32. Anon. (2008) Physical activity guidelines for Americans. Okla Nurse 53, 25.

33. Strong WB, Malina RM, Blimkie CJ et al. (2005) Evidence based physical activity for school-age youth. J Pediatr 146, 732-737.

34. Huybrechts I, Bornhorst C, Pala V et al. (2011) Evaluation of the Children's Eating Habits Questionnaire used in the IDEFICS study by relating urinary calcium and potassium to milk consumption frequencies among European children. Int J Obes (Lond) 35, Suppl. 1, S69-S78.

35. Lanfer A, Hebestreit A, Ahrens W et al. (2011) Reproducibility of food consumption frequencies derived from the Children's Eating Habits Questionnaire used in the IDEFICS study. Int J Obes (Lond) 35, Suppl. 1, S61-S68.

36. Bel-Serrat S, Mouratidou T, Pala V et al. (2014) Relative validity of the Children's Eating Habits Questionnaire-food frequency section among young European children: the IDEFICS Study. Public Health Nutr 17, 266-276.
37. Booth VM, Rowlands AV \& Dollman J (2015) Physical activity temporal trends among children and adolescents. J Sci Med Sport 18, 418-425.

38. Verbestel V, De Henauw S, Bammann K et al. (2015) Are context-specific measures of parental-reported physical activity and sedentary behaviour associated with accelerometer data in 2-9-year-old European children? Public Health Nutr 18, 860-868.

39. Mushtaq MU, Gull S, Mushtaq K et al. (2011) Dietary behaviors, physical activity and sedentary lifestyle associated with overweight and obesity, and their sociodemographic correlates, among Pakistani primary school children. Int J Behav Nutr Phys Act 8, 130.

40. Ranjit N, Evans MH, Byrd-Williams C et al. (2010) Dietary and activity correlates of sugar-sweetened beverage consumption among adolescents. Pediatrics 126, e754-e761.

41. Bammann K, Sioen I, Huybrechts I et al. (2011) The IDEFICS validation study on field methods for assessing physical activity and body composition in children: design and data collection. Int J Obes (Lond) 35, Suppl. 1, S79-S87.

42. Ojiambo $\mathrm{R}$, Cuthill $\mathrm{R}$, Budd $\mathrm{H}$ et al. (2011) Impact of methodological decisions on accelerometer outcome variables in young children. Int J Obes (Lond) 35, Suppl. 1, S98-S103.

43. Mouratidou T, Mesana MI, Manios Y et al. (2012) Assessment tools of energy balance-related behaviours used in European obesity prevention strategies: review of studies during preschool. Obes Rev 13, Suppl. 1, 42-55.

44. Bach-Faig A, Berry EM, Lairon D et al. (2011) Mediterranean diet pyramid today. Science and cultural updates. Public Health Nutr 14, 2274-2284.

45. Cole TJ, Flegal KM, Nicholls D et al. (2007) Body mass index cut offs to define thinness in children and adolescents: international survey. BMJ 335, 194. 